OPEN ACCESS

Edited by:

Fenglin Liu,

Fudan University, China

Reviewed by:

XiuJuan Qu,

The First Affiliated Hospital of China Medical University, China Dong-Qiu Dai,

China Medical University, China

${ }^{*}$ Correspondence:

Lin Chen

chenlin301plagh@sina.com Xinxin Wang

301wxx@sina.com

${ }^{\dagger}$ These authors share senior authorship

Specialty section: This article was submitted to Gastrointestinal Cancers, a section of the journal

Frontiers in Oncology

Received: 04 August 2020 Accepted: 05 January 2021 Published: 25 February 2021

Citation:

Xie T, Guo X, Wu D, Li S, LU Y, Wang $X$ and Chen L (2021) PAFAH1B3 Expression Is Correlated With Gastric Cancer Cell Proliferation and Immune Infiltration.

Front. Oncol. 11:591545. doi: 10.3389/fonc.2021.591545

\section{PAFAH1B3 Expression Is Correlated With Gastric Cancer Cell Proliferation and Immune Infiltration}

\author{
Tianyu Xie ${ }^{1,2}$, Xin Guo ${ }^{2}$, Di Wu ${ }^{2}$, Shuo $L^{1,2}$, Yixun $L^{2}{ }^{2}$, Xinxin Wang ${ }^{1,2 * t}$ and Lin Chen ${ }^{1,2 * t}$ \\ ${ }^{1}$ School of Medicine, Nankai University, Tianjin, China, ${ }^{2}$ Department of General Surgery, Chinese PLA General Hospital, \\ Beijing, China
}

Background: Platelet activating factor acetylhydrolase 1b catalytic subunit 3 (PAFAH1B3) is associated with a variety of human diseases. However, its function in gastric cancer remains uncertain.

Methods: PAFAH1B3 expression was analyzed in The Cancer Genome Atlas (TCGA) and genotype-tissue expression pan-cancer data. The association between PAFAH1B3 expression and patient prognosis was evaluated using TCGA clinical survival data. Enrichment analysis of PAFAH1B3 was performed using the clusterProfiler R software package. Moreover, the correlation between PAFAH1B3 expression and immune cell infiltration were evaluated by analyzing TCGA database. CCK8 assay and colonyformation assay were performed to assess the effect of PAFAH1B3 on the proliferation of gastric cancer cells. Transwell assay was used to evaluate the impact of PAFAH1B3 on gastric cancer cell migration. Western blot was performed to evaluate the role of PAFAH1B3 on signaling pathways in gastric cancer cells.

Results: PAFAH1B3 was highly expressed in many types of tumors including gastric cancer. High PAFAH1B3 expression was significantly correlated with proliferation-related gene sets involved in DNA replication, the cell cycle, and cell cycle checkpoints. Further analysis showed that high PAFAH1B3 expression was associated with high M1 macrophage and CD8-positive $T$ cell infiltration scores. PAFAH1B3 knockdown inhibited the proliferation, migration, and the activation of oncogenic signaling in gastric cancer cells.

Conclusions: Our findings suggest that PAFAH1B3 may be an oncogene in gastric cancer.

Keywords: PAFAH1B3, gastric cancer, cell proliferation, immune infiltration, oncogene

\section{INTRODUCTION}

Gastric cancer is a major malignant tumor of the digestive system. It is prone to relapse and metastasis during the late stage and has a high mortality rate (1). At present, there is a lack of effective early diagnostic markers to detect and treat gastric cancer. With the rapid development of high-throughput sequencing technology and transcriptomic research, more driver genes continue to be discovered. However, there remains an urgent need to identify more key driver oncogenes, especially those that affect the composition of the immune microenvironment in gastric cancer. 
Platelet activating factor acetylhydrolase $1 \mathrm{~b}$ catalytic subunit 3 (PAFAH1B3) is one of the catalytic subunits of Platelet-activating factor (PAF) acetylhydrolase, which was reported to play crucial roles in certain types of cancers by regulating PAF activity $(2,3)$. Previous studies have indicated the association between PAFAH1B3 and cancer progression. For example, PAFAH1B3 expression was found to be higher in hypopharyngeal squamous cell carcinoma tissues than in adjacent non-tumor samples and predicted a poor outcome (4). Selective inhibition of PAFAH1B3 has been reported to impair cancer cell survival (5). However, the role of PAFAH1B3 in gastric cancer remains unknown.

In this study, we evaluated PAFAH1B3 expression in various tumors in The Cancer Genome Atlas (TCGA) and its correlation with patient prognosis. We further examined the association of PAFAH1B3 expression with molecular pathways in gastric cancer. Since immune cell infiltration is important for the prognosis of gastric cancer patients $(6,7)$, we also examined the correlation between PAFAH1B3 expression and the immune cell infiltration scores. Finally, we examined the effect of PAFAH1B3 knockdown on the apoptosis and proliferation of gastric cancer cells. Our results offer novel insights into the functional role of PAFAH1B3 in gastric cancer.

\section{MATERIALS AND METHODS}

\section{Data Collection and Analysis}

PAFAH1B3 expression profiles and TCGA and Genotype-Tissue Expression (GTEx) clinical pan-cancer data were downloaded from the University of California, Santa Cruz (UCSC) Xena database (https://xenabrowser.net/datapages/), including data on adrenocortical carcinoma (ACC), bladder urothelial carcinoma (BLCA), breast invasive carcinoma (BRCA), cervical squamous cell carcinoma and endocervical adenocarcinoma (CESC), cholangiocarcinoma (CHOL), colon adenocarcinoma (COAD), esophageal carcinoma (ESCA), glioblastoma multiforme (GBM), head and neck squamous cell carcinoma (HNSC), kidney chromophobe, kidney renal clear cell carcinoma, kidney renal papillary cell carcinoma (KIRP), acute myeloid leukemia (LAML), brain lower grade glioma (LGG), liver hepatocellular carcinoma (LIHC), lung adenocarcinoma (LUAD), lung squamous cell carcinoma (LUSC), ovarian serous cystadenocarcinoma (OV), pancreatic adenocarcinoma (PAAD), prostate adenocarcinoma (PRAD), rectum adenocarcinoma (READ), skin cutaneous melanoma (SCKM), stomach adenocarcinoma (STAD), testicular germ cell tumor (TGCT), thyroid carcinoma (THCA), uterine corpus endometrial carcinoma (UCEC), and uterine carcinosarcoma (UCS). To evaluate PAFAH1B3 expression, tumor tissues were obtained from TCGA, and normal tissues were obtained from TCGA and the GTEx database.

\section{Correlation and Enrichment Analyses}

Pearson correlation analysis of PAFAH1B3 mRNA and other mRNAs was performed in gastric cancer using TCGA STAD data. The 300 genes most positively associated with PAFAH1B3 were selected for enrichment analysis to determine the function of PAFAH1B3. Gene ontology (GO) analysis was performed using the EnrichGO function in the clusterProfiler R software package $\mathrm{R}$ with the following parameters: ont $=$ all, pvalue-Cutoff $=0.05$, and qvalue-Cutoff $=0.05$. Gene set enrichment analysis was performed using the gseKEGG and gsePathway functions in clusterProfiler with the following parameters: $n$ Perm $=1,000$, $\min G S S i z e=10$, maxGSSize $=1,000$, and pvalue-Cutoff $=0.05$.

\section{Immune Cell Infiltration}

We downloaded pan-cancer immune cell infiltration scores from TCGA that were derived from a previously published study (8), the results of which were based on the CIBERSORT analytical tool (9). TCGA gastric cancer samples were divided into two groups according to the median PAFAH1B3 expression (high versus low level), and their immune cell infiltration levels were compared.

\section{Cell Culture and Treatment}

AGS, MGC-803, BGC-823, SGC-7901 cell lines were purchased from the Cell Bank of the Shanghai Institute of Cells, Chinese Academy of Science (Shanghai, China). All cell lines were cultured in Dulbecco's modified Eagle's medium (Gibco) in $10-\mathrm{cm}$ petri dish (Corning) at $37^{\circ} \mathrm{C}$ with $5 \% \mathrm{CO}_{2}$. Cell transfection was performed using the Lipofectamine 2000 reagent (Invitrogen) according to the manufacturer's protocol. Briefly, cells were seeded in six-well plates (Corning) and grown to a cell density of $30 \%$ and then transfected and cultured at $37^{\circ} \mathrm{C}$ for a further $48 \mathrm{~h}$, followed by harvesting for quantitative reverse-transcription polymerase chain reaction (qRT-PCR) and other experiments.

\section{RNA Extraction and qRT-PCR}

Total RNA from approximately $1 \times 10^{6}$ cells were isolated using TRIzol reagent (Pufei, Shanghai, China) according to the manufacturer's protocol. The primers used for qRT-PCR, including those for PAFAH1B3 and GAPDH, were obtained from Applied Biosystems (Ribo, Guangzhou, China). The primer sequences were (5'-3'): PAFAH1B3 forward -GAGAAGAACC GACAGGTGAAC, reverse-CGGCAAACAGGTGTGTAGC. GAPDH forward-CTGGGCTACACTGAGCACC, reverseAAGTGGTCGTTGAGGGCAATG. QRT-PCR parameters were: $95^{\circ} \mathrm{C} 6 \mathrm{~min}$; $\left(95^{\circ} \mathrm{C} 10 \mathrm{~s}, 58^{\circ} \mathrm{C} 30 \mathrm{~s}\right) \times 40$ amplification cycles. Relative expression levels were normalized to internal controls and calculated according to the $2-\Delta \Delta \mathrm{CT}$ method.

\section{Western Blotting}

Total protein from approximately $1 \times 10^{6}$ cells were extracted using radioimmunoprecipitation assay buffer and then quantified using the bicinchoninic acid method (Beyotime, Shanghai, China). Equal amounts of protein sample were separated using 8-15\% sodium dodecyl sulfate-polyacrylamide gel electrophoresis and then transferred to nitrocellulose membranes. Membranes were blocked with 5\% non-fat milk in TBST (Abcam, Cambridge, UK) for $1 \mathrm{~h}$ at room temperature and incubated with primary antibody (Abcam) at $4^{\circ} \mathrm{C}$ overnight. Membranes were then washed three times andincubated with horseradish peroxidase-labeled secondary antibody (Santa Cruz Biotechnology, Dallas, TX, USA) for $1 \mathrm{~h}$. Signals were detected 
using an enhanced Chemiluminescent Western Blot Analysis Kit (Thermo Fisher Scientific, Inc.).

\section{CCK8 Assay}

Cells in the logarithmic growth phase from each experimental group were trypsinized, resuspended in complete medium, and cultured overnight. On the second day, cell proliferation was evaluated by Cell Counting Kit- 8 reagent (Abcam) according to the manufacturer's protocol. Optical density values at $460 \mathrm{~nm}$ were detected using a microplate reader (Molecular Devices, Rockford, IL, USA).

\section{Colony-Formation Assay}

One-hundred gastric cancer cells were plated into six-well plates and cultured for 10 days. Cell colonies were fixed and stained with crystal violet (Beyotime, China) for $5 \mathrm{~min}$ in $10 \%$ ethanol. Cell colonies were imaged and counted.

\section{Transwell Assay}

Thirty thousand gastric cancer cells were plated in the upper chamber of the transwell chamber (Corning, USA) in a 24-well plate. $24 \mathrm{~h}$ after incubation at $37^{\circ} \mathrm{C}$, cells on the top surface of the chamber were removed by wiping. Cells on the bottom surface of the chamber were fixed with $4 \%$ paraformaldehyde for $10 \mathrm{~min}$ and stained with crystal violet (Beyotime, China) for $5 \mathrm{~min}$. The number of migrated cells were imaged and counted.

\section{Statistical Analyses}

Data are expressed as means \pm standard deviation. All data were analyzed using SPSS ver. 21.0 software (SPSS, Inc., Chicago, IL, USA). The normality of the data was tested using the Kolmogorov-Smirnov test. Pairwise differences between groups were analyzed using Student's $t$-test. Significance was assessed at a level of $\mathrm{P}<0.05$.

A

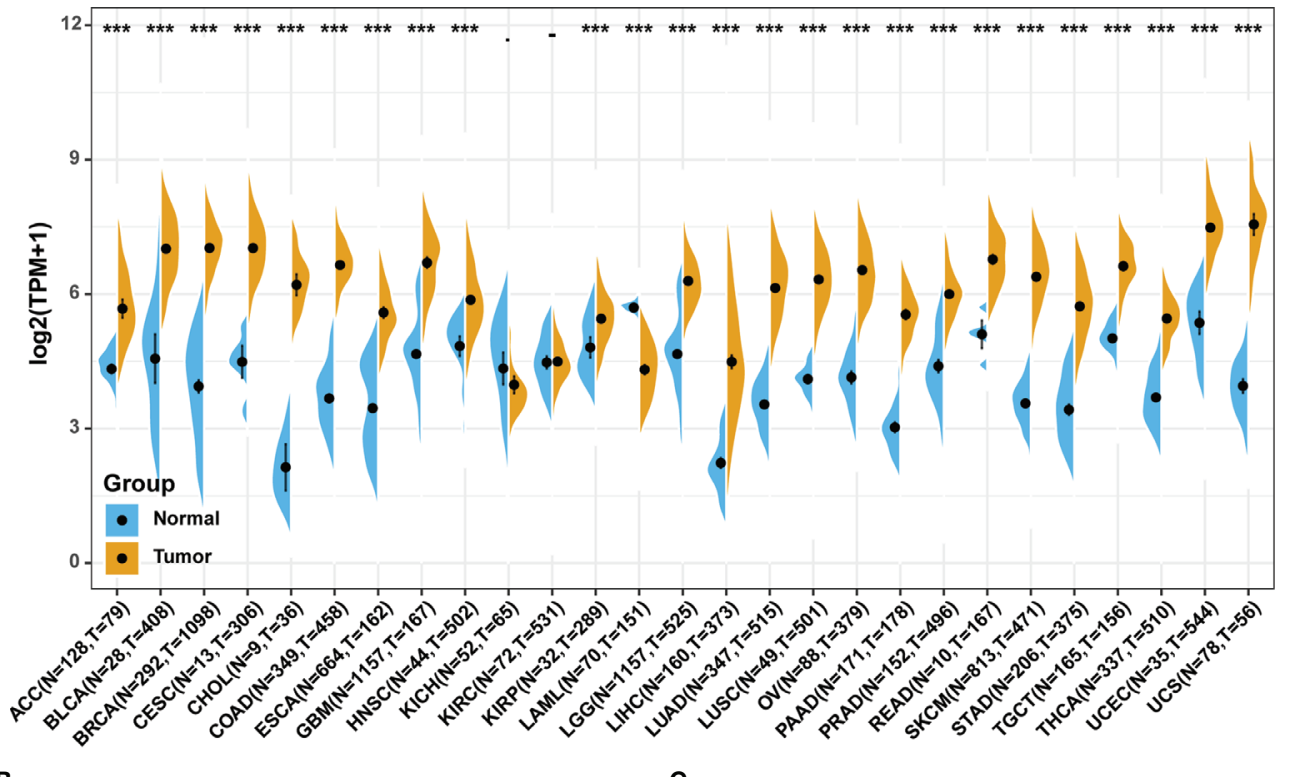

B

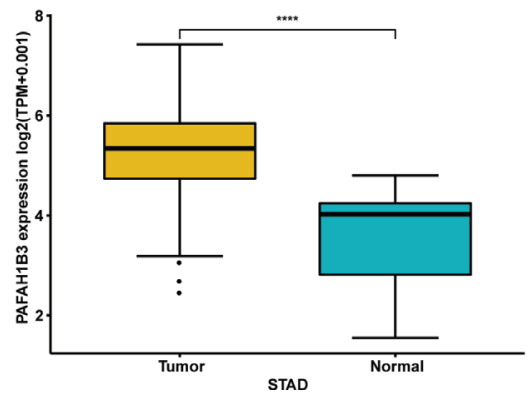

C

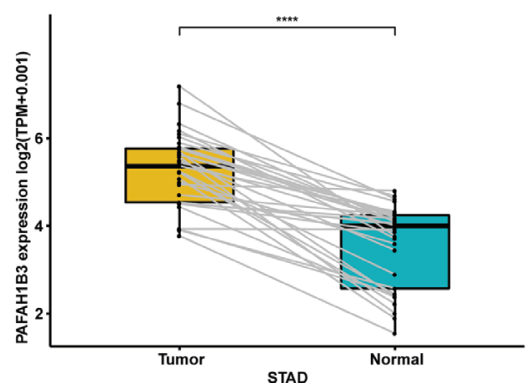

FIGURE 1 | Pan-cancer PAFAH1B3 expression analysis. (A) PAFAH1B3 expression in tumor and normal tissues in pan-cancer data of The Cancer Genome Atlas (TCGA) and GTEx. (B) PAFAH1B3 expression in tumor and normal tissues in STAD from TCGA. (C) PAFAH1B3 expression in paired tumor and normal tissues in STAD from TCGA. Data were shown as mean $\pm S D$. ${ }^{\star} p<0.05,{ }^{* \star} p<0.01,{ }^{* \star *} p<0.001,{ }^{* * \star *} p<0.0001$. 


\section{RESULTS}

\section{Pan-Cancer PAFAH1B3 Expression Analysis}

We first evaluated PAFAH1B3 expression in TCGA and GTEx pan-cancer database, and found higher PAFAH1B3 expression in 24 tumors compared with the corresponding normal tissues, including: ACC, BLCA, BRCA, CESC, CHOL, COAD, ESCA, GBM, HNSC, KIRP, LGG, LIHC, LUAD, LUSC, OV, PAAD, PRAD, READ, SCKM, STAD, TGCT, THCA, UCEC, and UCS (Figure 1A).

Particularly, high PAFAH1B3 expression was observed in STAD gastric cancer in the TCGA cohort (Figure 1B) compared with the adjacent tissues (Figure 1C), suggesting that PAFAH1B3 may play a role in the pathogenesis of gastric cancer.

\section{Association Between PAFAH1B3 Expression and Cancer Patient Prognosis}

To evaluate the utility of PAFAH1B3 expression in predicting cancer patient prognosis, we analyzed the association between PAFAH1B3 expression and overall survival in the TCGA cohort. The results showed that higher PAFAH1B3 expression was significantly associated with poor prognosis in ACC $(\mathrm{P}=$ $0.00023)$, LIHC $(\mathrm{P}=0.0011)$, LUAD $(\mathrm{P}=0.015)$, MESO $(\mathrm{P}=$
0.003), SARC $(\mathrm{P}=0.011)$, and SKCM $(\mathrm{P}=0.0033)$ (Figures 2A-F), indicating that PAFAH1B3 is a potential oncogene in these types of cancers.

\section{Correlation and Enrichment Analyses}

To further explore the functions and pathways affected by PAFAH1B3, we performed correlation analyses between PAFAH1B3 and all other mRNAs in gastric cancer using TCGA data. The 300 genes most positively associated with PAFAH1B3 were selected for enrichment analysis, and the top 50 genes were displayed in a heatmap (Figure 3). We further explored potential functional pathways based on the top 300 genes using the R software clusterProfiler package. GO functional enrichment analysis revealed that PAFAH1B3 was associated mainly with cell proliferation-related pathways including DNA replication and cell cycle checkpoints (Figures $4 \mathbf{A}-\mathbf{C}$ ). Gene set enrichment analysis was conducted to search the Kyoto Encyclopedia of Genes and Genomes (KEGG) and Reactome pathway databases. The KEGG analysis results showed that spliceosome, ribosome, and cell cycle terms were significantly enriched (Figure 4D). The Reactome analysis revealed significant enrichment of cell cycle, $M$ phase, and DNA repair pathways (Figure 4E). These results suggest that high PAFAH1B3 expression is associated with the hyperactivation
A

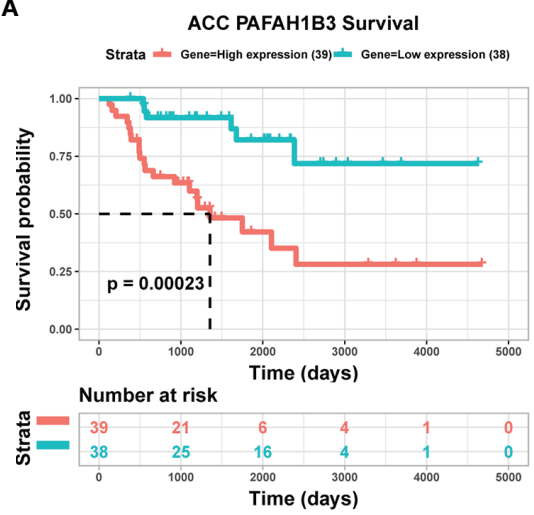

D
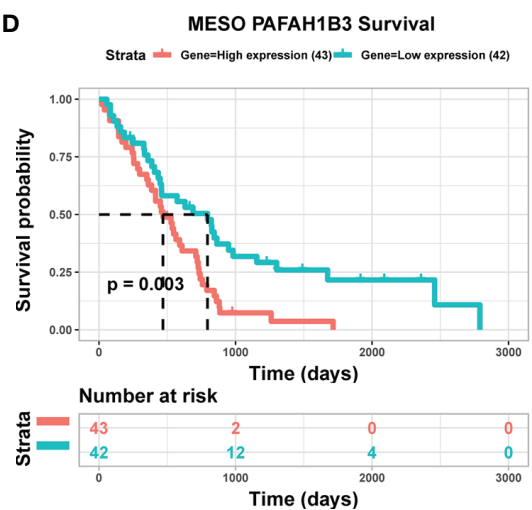

B
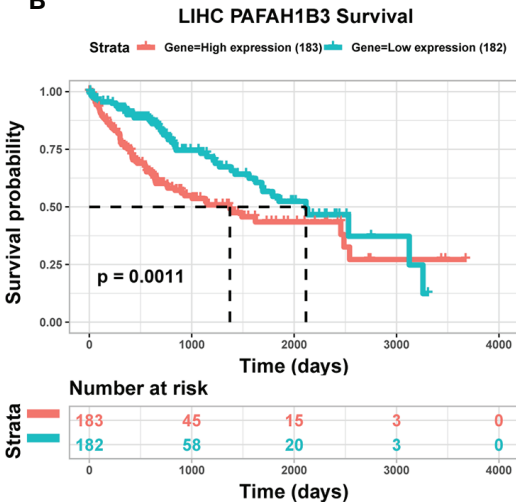

E

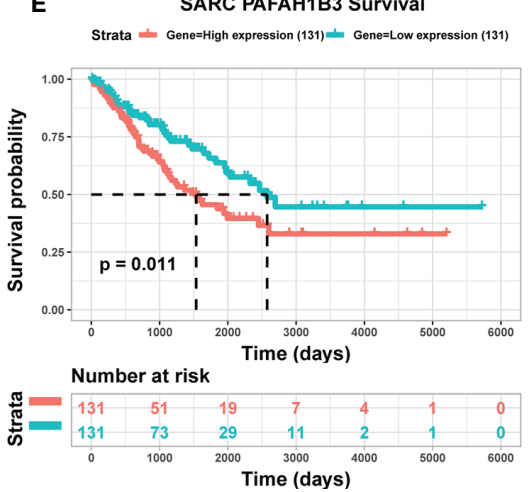

C

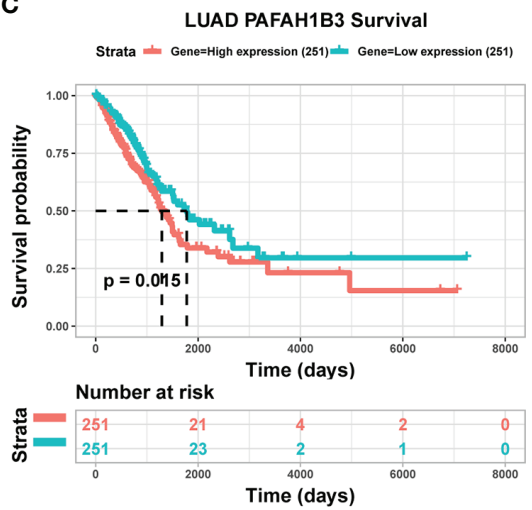

$\mathbf{F}$

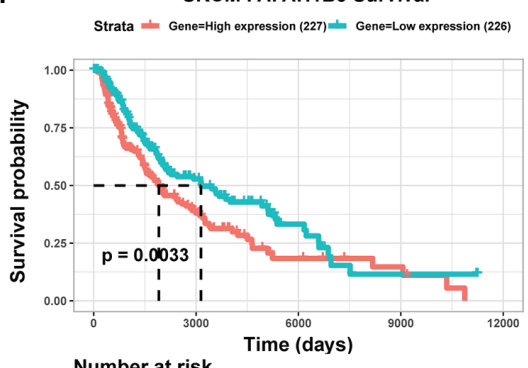

FIGURE 2 | The association between PAFAH1B3 expression and cancer patient prognosis. (A-F) The correlation between PAFAH1B3 expression and the prognosis of various cancer types was analyzed using The Cancer Genome Atlas (TCGA) database. 


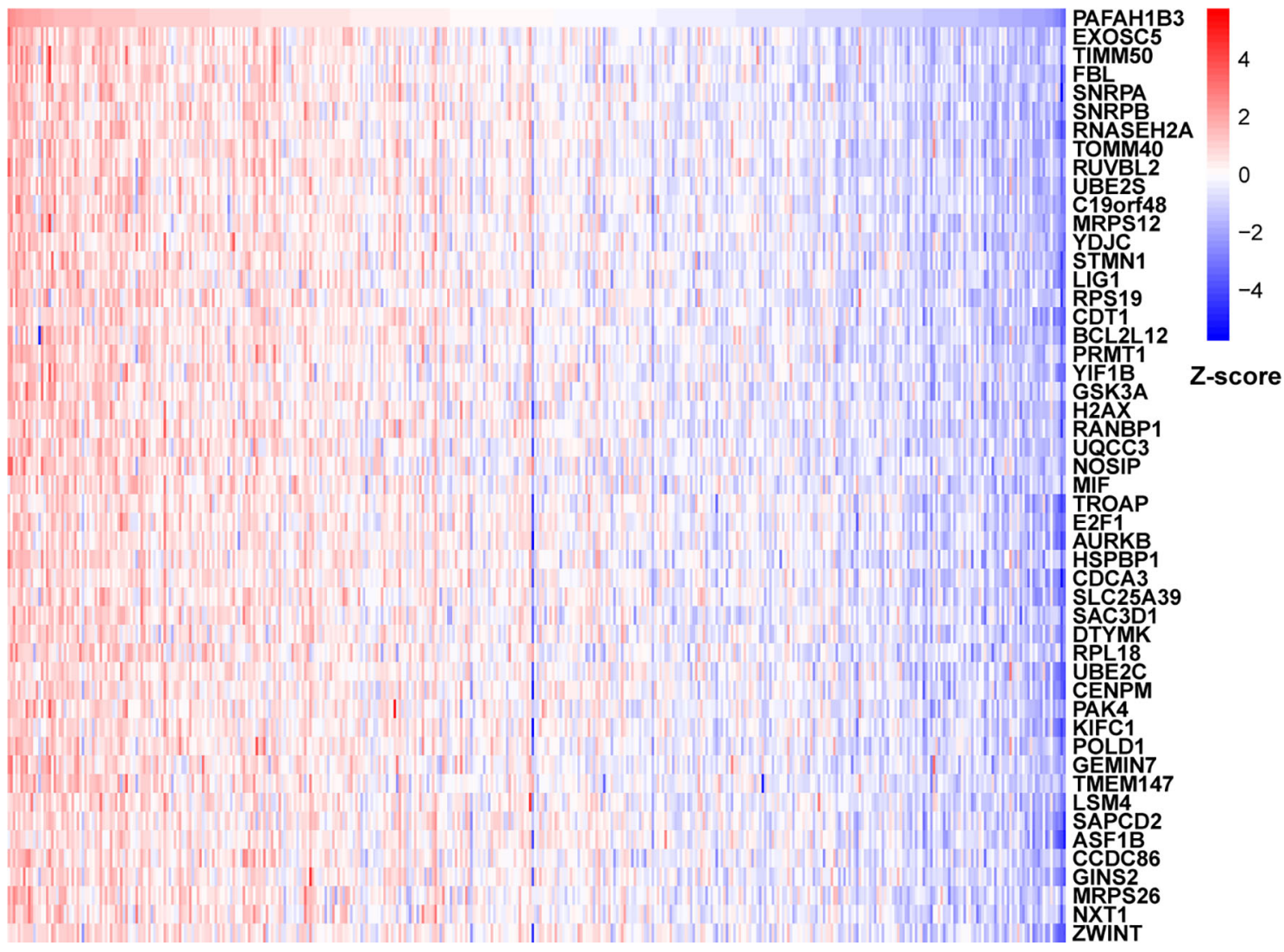

FIGURE 3 | The correlation analysis of PAFAH1B3. Top 50 genes most positively associated with PAFAH1B3 were shown in heatmap. Data were normalized by Zscore standardization method.

of multiple oncogenic pathways in gastric cancer, especially signalings which control cell proliferation.

\section{Correlation Between Immune Cell Infiltration and PAFAH1B3 Expression}

Next, we analyzed the immune cell infiltration scores of gastric cancer patients from TCGA database. The infiltration scores of M1 macrophages and the CD8-positive T cells were higher in the high-PAFAH1B3-expression cohort compared with those in the low-PAFAH1B3-expression cohort (Figure 5A).

PAFAH1B3 expression was significantly positively correlated with the M1 macrophage infiltration and CD8-positive T cell infiltration (Figure 5B), indicating that high PAFAH1B3 expression promotes the intratumoral accumulation of CD8positive $\mathrm{T}$ cells and macrophages, especially M1-like macrophages. These results suggest that high PAFAH1B3 expression is closely related to the immune-activated status of gastric cancer.

\section{PAFAH1B3 Knockdown Inhibited the Malignant Behaviors and Signaling in Gastric Cancer Cells}

Next, we evaluated PAFAH1B3 expression in multiple gastric cancer cell lines, and found relatively higher expression of
PAFAH1B3 in BGS-823 and SGC-7901 cells compared with that in AGS and MGS-803 cells (Figure 6A). To explore the biological effects of PAFAH1B3 on gastric cancer cell proliferation, we knocked down PAFAH1B3 expression in BGS-823 and SGC-7901 cells via two PAFAH1B3 shRNA, and validated the successful silence of PAFAH1B3 expression in these two cell lines (Figure 6B). Next, we conducted CCK8 assay to evaluate cell proliferation. The Results showed that the proliferation rates of the BGS-823 and SGC-7901 cells were significantly inhibited following PAFAH1B3 knockdown (Figure 6C). The negative effect of PAFAH1B3 on gastric cancer cell proliferation was further confirmed by colony-formation assay (Figure 6D). Besides, PAFAH1B3 silencing also significantly impaired the migratory ability of gastric cancer cells as evidenced by Transwell assay (Figure 6E). Finally, we looked at the impact of PAFAH1B3 on several oncogenic signaling pathways by Western blot. The results showed that PAFAH1B3 knockdown in SGC-7901 cells led to the obviously downregulated levels of IRS1, Myc, HMGB1, and PTGS2. On the other hand, the levels of MET and FOXM1 were slightly upregulated. The level of EZH2 was unchanged by PAFAH1B3 knockdown (Figure 6F). Taken together, PAFAH1B3 facilitates the proliferation, migration, and the activation of oncogenic signaling in gastric cancer cells. 


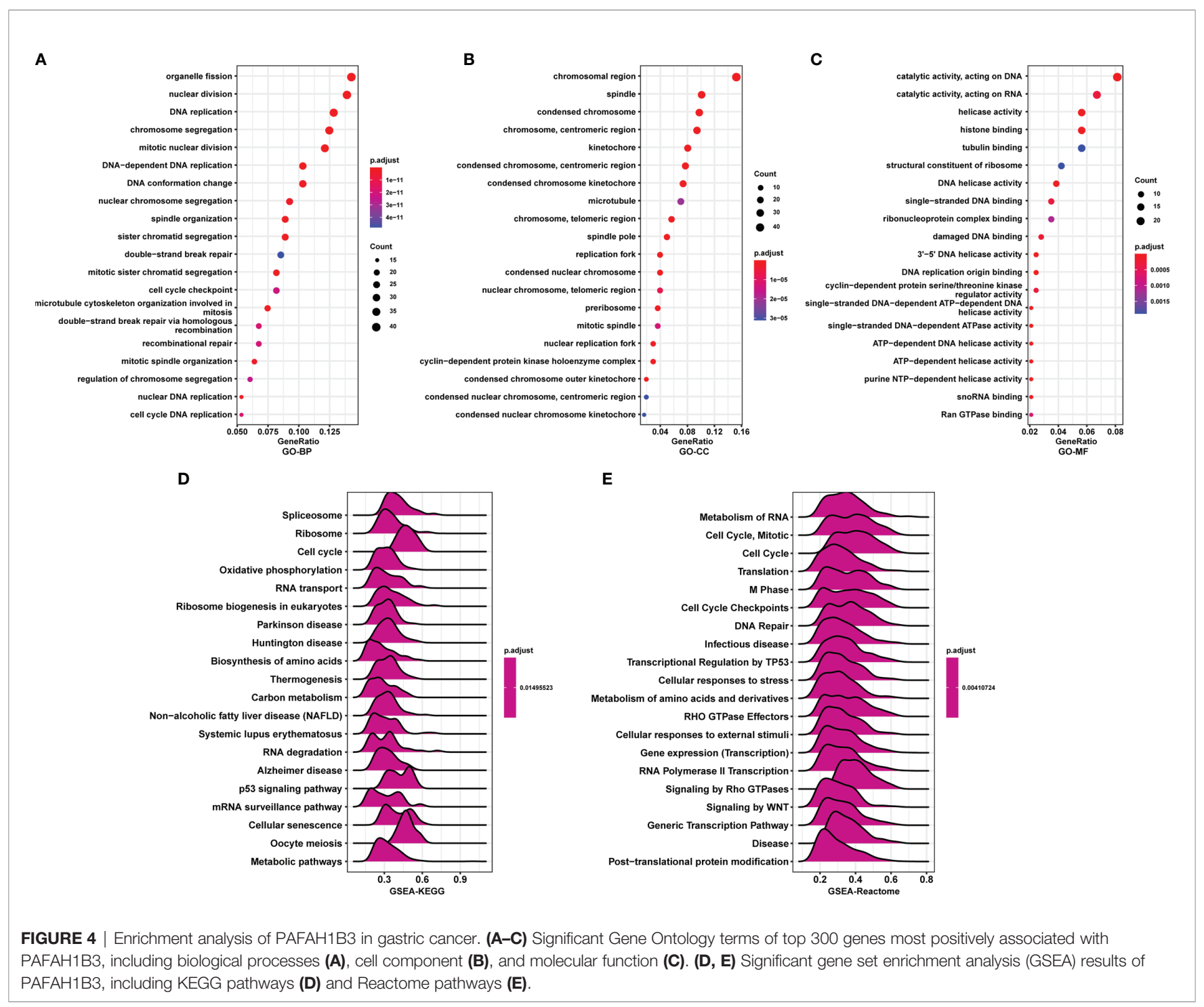

\section{DISCUSSION}

In recent years, $\mathrm{PAFAH} 1 \mathrm{~B} 3$ was reported to play regulatory roles in various kinds of diseases, including human cancers. For example, in hypopharyngeal squamous cell carcinoma (HSCC), the expression of PAFAH1B3 was reported to be upregulated and was correlated with poor patient prognosis. Silencing PAFAH1B3 expression restricted the proliferation, invasion, and migration of HSCC cells (4). Metabolomic study indicated that inhibition of PAFAH1B3 suppressed the growth of breast cancer by upregulating the levels of anti-tumor lipids, such as ceramides and several PPAR $\alpha$ ligands, indicating that PAFAH1B3 serves as a metabolic oncogene in breast cancer (10). Similarly, selective small-molecule PAFAH1b3 inhibitors impaired the growth of neuroblastoma cells, suggesting that PAFAH1b3 might become a targetable oncoprotein (5). Nevertheless, the expression pattern of PAFAH1B3 in various human cancers and its prognostic values are still elusive. Here, by data mining using TCGA datasets, the hyperexpression of PAFAH1B3 was observed in 24 types of tumor tissues compared with normal tissues, suggesting that PAFAH1B3 might serve as a novel oncogene in these tumor types.

Next, we examined the expression level of PAFAH1B3 and its prognostic value using pan-cancer TCGA and GTEx data obtained from the UCSC Xena database. Compared with normal tissues, we found that PAFAH1B3 was highly expressed in ACC, BLCA, BRCA, CESC, CHOL, COAD, ESCA, GBM, HNSC, KIRP, LGG, LIHC, LUAD, LUSC, OV, PAAD, PRAD, READ, SCKM, STAD, TGCT, THCA, UCEC, and UCS, whereas its expression was low in LAML tissue. PAFAH1B3 was particularly highly expressed in gastric cancer tissues compared with adjacent normal tissues according to TCGA data. Overexpression of PAFAH1B3 generally predicts a poor prognosis in ACC, LIHC, LUAD, MESO, SARC, and 
A

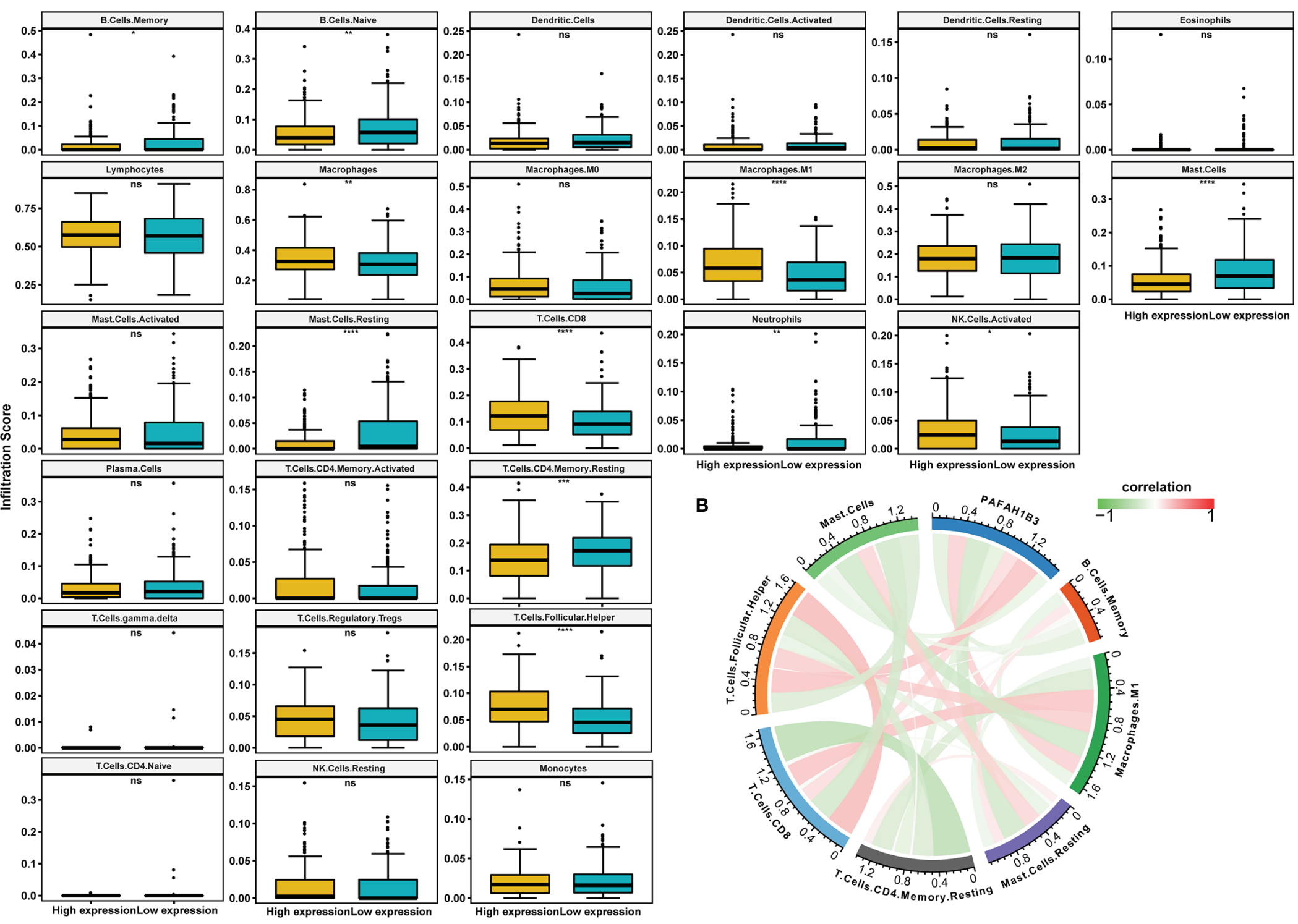

FIGURE 5 | The correlation analysis between immune cell infiltration and PAFAH1B3 in gastric cancer. (A) The immune cell infiltration level in high and low PAFAH1B3 expression groups in gastric cancer of The Cancer Genome Atlas (TCGA) cohort. (B) The correlation between PAFAH1B3 and the immune cell infiltration levels, red represents positive correlation, green represents negative correlation, and the deeper the color, the stronger the correlation. Data were shown as mean $\pm \mathrm{SD} .{ }^{*} \mathrm{p}<0.05,{ }^{* \star} \mathrm{p}<0.01,{ }^{* \star *} \mathrm{p}<0.001,{ }^{\star \star \star *} \mathrm{p}<0.0001$. ns, no significance. 
A

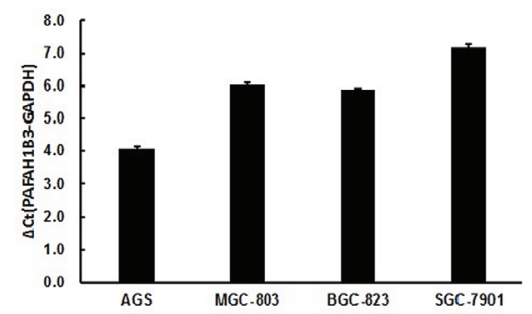

C

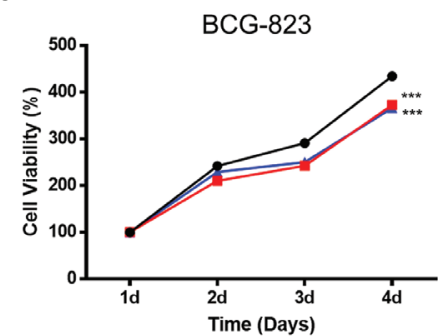

D
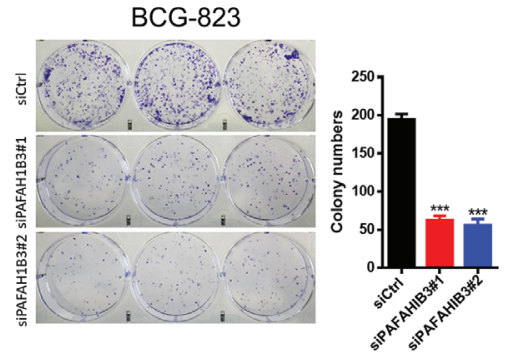

E

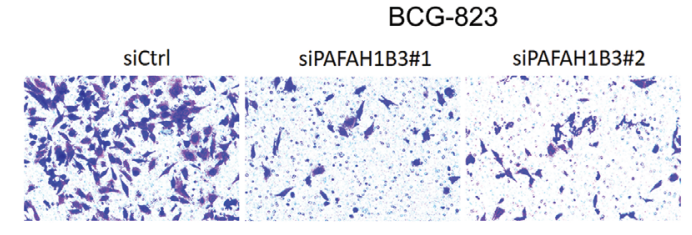

SGC-7901

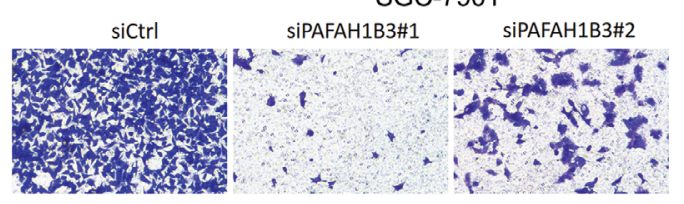

B
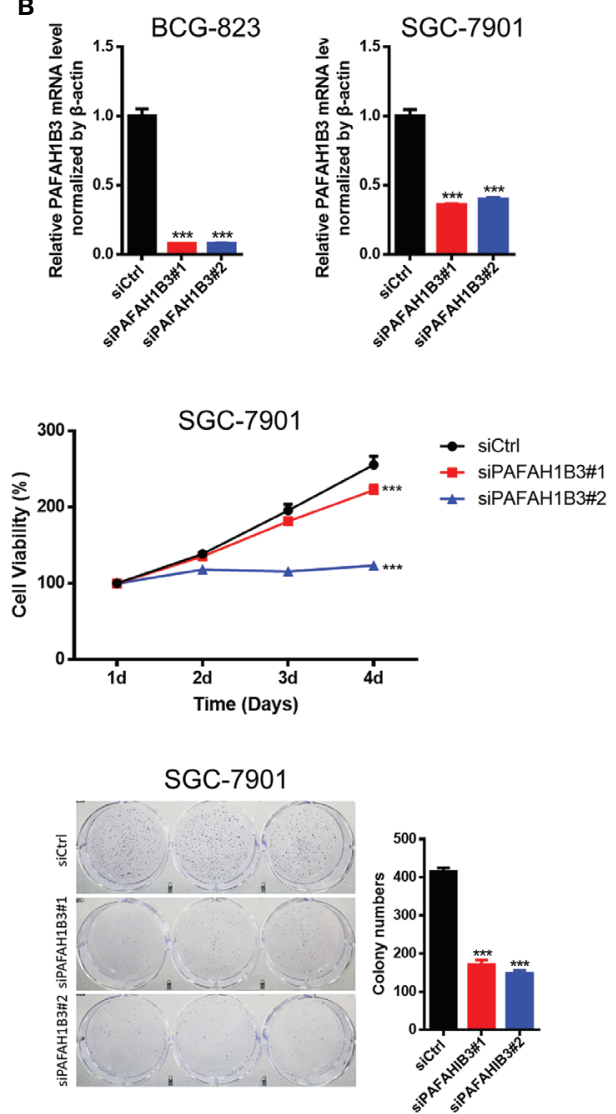

F
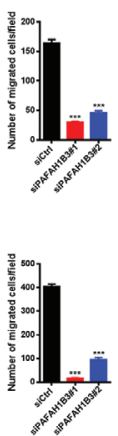

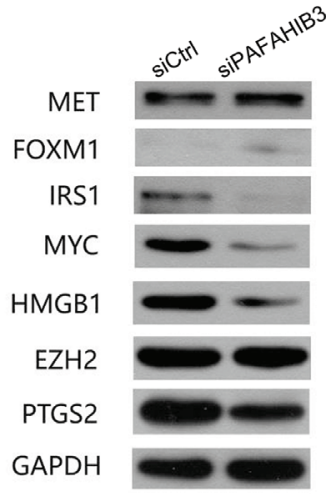

FIGURE 6 | Knockdown of PAFAH1B3 inhibited the proliferation and migration of gastric cancer cells. (A) The level of PAFAH1B3 was evaluated in various gastric cancer cell lines by qRT-PCR. (B) BCG-823 and SGC-7901 cells were transfected with si-PAFAH1B3, the level of PAFAH1B3 was evaluated by qRT-PCR. (C, D) The proliferation of gastric cells (GC) was examined by CCK-8 assay (C) and colony-formation assay. (E) The migration of GC cells was examined by transwell assay. (F) The expression levels of indicated proteins were evaluated by Western blot in control and PAFAH1B3-silenced BCG-823 cells. Data were shown as mean $\pm \mathrm{SD} .{ }^{* \star *} \mathrm{p}<0.001$.

SKCM. These results support the use of PAFAH1B3 as a prognostic biomarker of tumor prognosis.

Our enrichment analysis results showed that PAFAH1B3 is closely related to cell proliferation-related pathways such as DNA replication, the cell cycle, and cell cycle checkpoints. Further analyses demonstrated that PAFAH1B3 knockdown inhibited the proliferation of the AGS and MGC- 803 gastric cancer cell lines. On the other hand, the signaling mechanisms underlying the function of PAFAH1B3 in tumor cells are largely unknown to date. Here, we found that PAFAH1B3 knockdown in gastric cancer cells downregulated the levels of IRS1, Myc, HMGB1, and PTGS2, which were reported to serve as oncogenes 
in many types of cancers (11-14). These results suggest that PAFAH1B3 facilitated the activation of multiple oncogenic signaling pathways in gastric cancer cells.

Stroma cells in tumor microenvironment, especially immune cells, are vital elements which have profound impacts on regulating the malignant behaviors of tumor cells (15-17). Accumulating evidence has elucidated their clinicopathological significance in predicting the outcomes and therapeutic efficacy in cancer patients $(18,19)$. The infiltration of CD8positive $\mathrm{T}$ cells and tumor-associated macrophages was reported to facilitate the progression of epithelial gastric cancer (20-22). In the present study, infiltration levels of CD8-positive T cells and M1 macrophages were significantly higher in the gastric cancer group with high PAFAH1B3 expression. Moreover, positive correlations were detected between PAFAH1B3 expression and infiltration levels of CD8-positive T cells and M1 macrophages, indicating a key role of PAFAH1B3 in regulating tumor immunology.

In summary, PAFAH1B3 may play a pathogenic role in gastric cancer progression, both acting on tumor cells or on tumor-infiltrating immune cells.

\section{REFERENCES}

1. Guggenheim DE, Shah MA. Gastric cancer epidemiology and risk factors. J Surg Oncol (2013) 107(3):230-6. doi: 10.1002/jso.23262

2. Satoh K, Imaizumi T, Kawamura Y, Yoshida H, Hiramoto M, Takamatsu S, et al. Platelet-activating factor (PAF) stimulates the production of PAF acetylhydrolase by the human hepatoma cell line, HepG2. J Clin Invest (1991) 87(2):476-81. doi: 10.1172/JCI115020

3. Heon Seo K, Ko HM, Kim HA, Choi JH, Jun Park S, Kim KJ, et al. Plateletactivating factor induces up-regulation of antiapoptotic factors in a melanoma cell line through nuclear factor-kappaB activation. Cancer Res (2006) 66 (9):4681-6. doi: 10.1158/0008-5472.CAN-05-3186

4. Xu J, Zang Y, Cao S, Lei D, Pan X. Aberrant expression of PAFAH1B3 associates with poor prognosis and affects proliferation and aggressiveness in hypopharyngeal squamous cell carcinoma. Onco Targets Ther (2019) 12:2799-808. doi: 10.2147/OTT.S196324

5. Chang JW, Zuhl AM, Speers AE, Niessen S, Brown SJ, Mulvihill MM, et al. Selective inhibitor of platelet-activating factor acetylhydrolases $1 \mathrm{~b} 2$ and $1 \mathrm{~b} 3$ that impairs cancer cell survival. ACS Chem Biol (2015) 10(4):925-32. doi: $10.1021 /$ cb500893q

6. Kumagai S, Togashi Y, Sakai C, Kawazoe A, Kawazu M, Ueno T, et al. An Oncogenic Alteration Creates a Microenvironment that Promotes Tumor Progression by Conferring a Metabolic Advantage to Regulatory T Cells. Immunity (2020) 53(1):187-203 e188. doi: 10.1016/j.immuni. 2020.06.016

7. Hirahara N, Tajima Y, Matsubara T, Fujii Y, Kaji S, Kawabata Y, et al. Systemic Immune-Inflammation Index Predicts Overall Survival in Patients with Gastric Cancer: a Propensity Score-Matched Analysis. J Gastrointest Surg (2020). doi: 10.1007/s11605-020-04710-7

8. Thorsson V, Gibbs DL, Brown SD, Wolf D, Bortone DS, Ou Yang TH, et al. The Immune Landscape of Cancer. Immunity (2018) 48(4):812-830 e814. doi: 10.1016/j.immuni.2018.03.023

9. Newman AM, Liu CL, Green MR, Gentles AJ, Feng W, Xu Y, et al. Robust enumeration of cell subsets from tissue expression profiles. Nat Methods (2015) 12(5):453-7. doi: 10.1038/nmeth.3337

10. Mulvihill MM, Benjamin DI, Ji X, Le Scolan E, Louie SM, Shieh A, et al. Metabolic profiling reveals PAFAH1B3 as a critical driver of breast cancer pathogenicity. Chem Biol (2014) 21(7):831-40. doi: 10.1016/ j.chembiol.2014.05.008

\section{DATA AVAILABILITY STATEMENT}

The datasets presented in this study can be found in online repositories. The names of the repository/repositories and accession number(s) can be found in the article/ supplementary material.

\section{AUTHOR CONTRIBUTIONS}

LC and XW designed the research. TX, DW, and SL performed the research and collected the data. XG and YL analyzed the data. TX drafted the manuscript. All authors contributed to the article and approved the submitted version.

\section{FUNDING}

National Defense Science and Technology Strategy Pilot program No. 19-ZLXD-12-35-03-600-01; Clinical Research Support Fund of PLA General Hospital No 2019XXJSYX07.

11. Chen H, Liu H, Qing G. Targeting oncogenic Myc as a strategy for cancer treatment. Signal Transduct Target Ther (2018) 3:5. doi: 10.1038/s41392-0180008-7

12. Williams CS, Mann M, DuBois RN. The role of cyclooxygenases in inflammation, cancer, and development. Oncogene (1999) 18(55):7908-16. doi: 10.1038/sj.onc.1203286

13. Tripathi A, Shrinet K, Kumar A. HMGB1 protein as a novel target for cancer. Toxicol Rep (2019) 6:253-61. doi: 10.1016/j.toxrep.2019.03.002

14. Reiss K, Del Valle L, Lassak A, Trojanek J. Nuclear IRS-1 and cancer. J Cell Physiol (2012) 227(8):2992-3000. doi: 10.1002/jcp.24019

15. Vilarino N, Bruna J, Bosch-Barrera J, Valiente M, Nadal E. Immunotherapy in NSCLC patients with brain metastases. Understanding brain tumor microenvironment and dissecting outcomes from immune checkpoint blockade in the clinic. Cancer Treat Rev (2020) 89:102067. doi: 10.1016/ j.ctrv.2020.102067

16. Sahin Ozkan H, Ugurlu MU, Yumuk PF, Kaya H. Prognostic Role of Immune Markers in Triple Negative Breast Carcinoma. Pathol Oncol Res (2020) 26 (4):2733-45. doi: 10.1007/s12253-020-00874-4

17. Binnewies M, Roberts EW, Kersten K, Chan V, Fearon DF, Merad M, et al. Understanding the tumor immune microenvironment (TIME) for effective therapy. Nat Med (2018) 24(5):541-50. doi: 10.1038/s41591-018-0014-x

18. Li C, Jiang $\mathrm{P}$, Wei $\mathrm{S}, \mathrm{Xu} \mathrm{X}$, Wang J. Regulatory $\mathrm{T}$ cells in tumor microenvironment: new mechanisms, potential therapeutic strategies and future prospects. Mol Cancer (2020) 19(1):116. doi: 10.1186/s12943-02001234-1

19. Erin N, Grahovac J, Brozovic A, Efferth T. Tumor microenvironment and epithelial mesenchymal transition as targets to overcome tumor multidrug resistance. Drug Resist Update (2020) 53:100715. doi: 10.1016/ j.drup.2020.100715

20. Zhang H, Li R, Cao Y, Gu Y, Lin C, Liu X, et al. Poor Clinical Outcomes and Immunoevasive Contexture in Intratumoral IL-10-Producing Macrophages Enriched Gastric Cancer Patients. Ann Surg (2020). doi: 10.1097/ SLA.0000000000004037

21. Liu Q, Yang C, Wang S, Shi D, Wei C, Song J, et al. Wnt5a-induced M2 polarization of tumor-associated macrophages via IL-10 promotes colorectal cancer progression. Cell Commun Signal (2020) 18(1):51. doi: 10.1186/ s12964-020-00557-2

22. Wang Z, Yang Y, Cui Y, Wang C, Lai Z, Li Y, et al. Tumor-associated macrophages regulate gastric cancer cell invasion and metastasis through 
TGFbeta2/NF-kappaB/Kindlin-2 axis. Chin J Cancer Res (2020) 32(1):72-88. doi: $10.21147 /$ j.issn.1000-9604.2020.01.09

Conflict of Interest: The authors declare that the research was conducted in the absence of any commercial or financial relationships that could be construed as a potential conflict of interest.
Copyright (๑) 2021 Xie, Guo, Wu, Li, Lu, Wang and Chen. This is an open-access article distributed under the terms of the Creative Commons Attribution License (CC BY). The use, distribution or reproduction in other forums is permitted, provided the original author(s) and the copyright owner(s) are credited and that the original publication in this journal is cited, in accordance with accepted academic practice. No use, distribution or reproduction is permitted which does not comply with these terms. 